\title{
OPTIMIZATION OF SPIRAL INDUCTOR ON SILICON
}

\author{
Arokiaswami Alphones *, Wong Kai Yee ^ \\ * School of Electrical \& Electronic Engineering, Nanyang Technological University, Singapore 639798 \\ ealphones@ntu.edu.sg
}

I Department of ECE, National University of Singapore, Singapore 117576

\begin{abstract}
A simulation program is developed based on Grover's formulas and Greenhouse method to calculate the inductance value and Q-factor for particular dimension and width distribution of spiral inductors. This program changes the width distribution through a predetermined number of quadratic distributions in an attempt to reduce energy losses and improve Q-factor. The result of this program provides a higher Q-factor together with the possibility of reducing the overall dimension of square spiral inductors as compared with conventional designs. The coordinates of dimension for the simulated inductor are also calculated enabling ease of verification and fabrication. Finally, the calculated results are verified by simulating the inductor using HPADS momentum which is MOM based electromagnetic simulator. The ease of use and rapid results of this program can find its attractiveness in wireless communications and related areas.
\end{abstract}

\section{INTRODUCTION}

The wireless environment of the future will require low cost and low power Si ICs. This will ultimately require higher levels of integration placing low-noise sensitive RF blocks on the same substrate. Due to the finite conductivity of the substrate, every device will be coupled to every other device on the IC.

Historically, inductors in silicon technology have low Q-factor resulting from the relatively high conductivity of typical silicon substrates. In addition, the ohmic losses of these structures are high because of the relatively thin $\mathrm{Al}$-based metallization employed in most VLSI processes. Therefore, in order to identify the optimal inductor layouts efficiently and account for the inductors and their parasitic in circuit simulation, an accurate equivalent circuit model is necessary [1]. Based on the insight from this model, a method is developed to estimate the optimal layout parameters with higher Q-factor of an inductor with specific requirements.

\section{UNIFORM SPIRAL INDUCTOR}

The physical model of a spiral inductor is shown in Figure 1. As inductance of square spiral inductors is a complicated function of geometry, the first step to the problem is to develop a systematic approach to calculate the length dimensions. Instead of dealing the square spiral as a whole, it is being breakdown into vertical and horizontal axes of reference. The length and negative pitch follows the system of axes, which set the basis for calculation of self-inductance, positive and negative mutual inductance $[2,3]$.

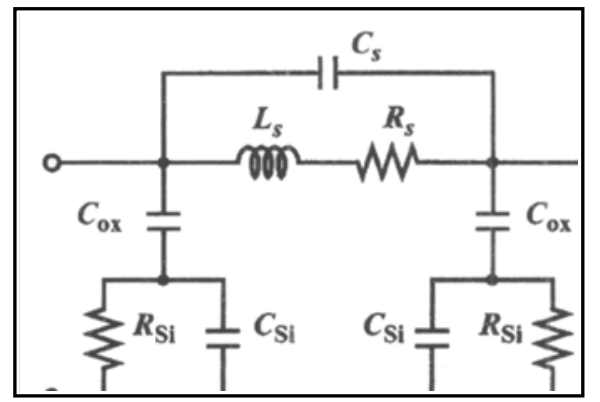

Figure 1: Lumped physical model of spiral inductor on silicon.

The program then proceeds to evaluate the frequency independent and dependent terms, which finally calculate the value of inductance, Q-factor and center frequency for the uniform spiral inductor. As the coordinates are calculated in the program, further verification is carried out using HPADS momentum to determine the accuracy of the circuit approach. Flowchart 1 shows the algorithm approach.

Table 1 shows the simulation results for both the circuit and HPADS simulation. The parameters $\{\mathrm{N}=7$, $\mathrm{w}=13 \mu \mathrm{m}, \mathrm{s}=7 \mu \mathrm{m}$, dout $=300 \mu \mathrm{m}\}$ that were set are based on [1] and this set the basis for comparison between some known results and the simulated results. Comparing the simulated results with that reported in literature, we can observe that the inductance value decreased by approximately $8 \%$ for the circuit model and increased by $7.7 \%$ for HPADS. Other values of comparison are not calculated since they are not stated explicitly in the reference.

\begin{tabular}{||c|c|c|c||}
\hline & $\begin{array}{c}\text { Q- } \\
\text { factor }\end{array}$ & Ls(nH) & Freq. $(\mathrm{GHz})$ \\
\hline $\begin{array}{c}\text { CIRCUIT } \\
\text { SIMULATION }\end{array}$ & 4.0919 & 7.3627 & 2.07 \\
\hline HPADS & 3.992 & 8.617 & 1.350 \\
\hline
\end{tabular}

Table 1: Simulation results for uniform spiral inductor. 


\section{VARIABLE SPIRAL INDUCTOR}

After the program's accuracy is verified, an optimization procedure is implemented to achieve higher Q-factor. The influence of magnetically induced losses is more significant in the inner turns of the coil where the magnetic field reaches its maximum. On the other hand, ohmic losses decrease with increasing width. It has been proposed using narrow width in the inner turns and broader width in the outer turns can achieve higher Q-factor [4]. However, the process discussed is random and time consuming.

Thus, in the second part of the program, the widths are varied between linear and quadratic distribution from the inner to outer turns in an attempt to achieve higher Q-factor and to find the optimal design layout and width distribution parameters. The equation governing the distribution of width is given below. "Vwidth" is the width size at each different turns numbered as " $x$ ". "Power" is the term controlling the type of distribution (Linear to Quadratic) and " $y_{\text {intercept" provide the starting }}$ width of the outmost turn and all other turns are computed based on this starting width.

$V_{\text {width }}=\left(\frac{x}{\text { gradient }}\right)^{\frac{1}{\text { power }}}+y_{\text {intercept }}$

where $\mathrm{x}=1, \ldots, \mathrm{N}$ (number of turns)

In Figure 2, the lines show the way the widths of spiral inductor are distributed according to the above equation. The $\mathrm{x}$-axis is an index system indicating the turn of the spiral inductor discussed with 1 to 7 representing outermost to innermost turn respectively. The y-axis indicates the width at that turn. The blue lines represent linear width distribution with "Power" = 1 while quadratic width distributions are red lines with "Power" $=2$ and green lines with "Power"= 3 . While keeping "Power" constant, "yintercept" is varied thus changing the gradient of the lines.

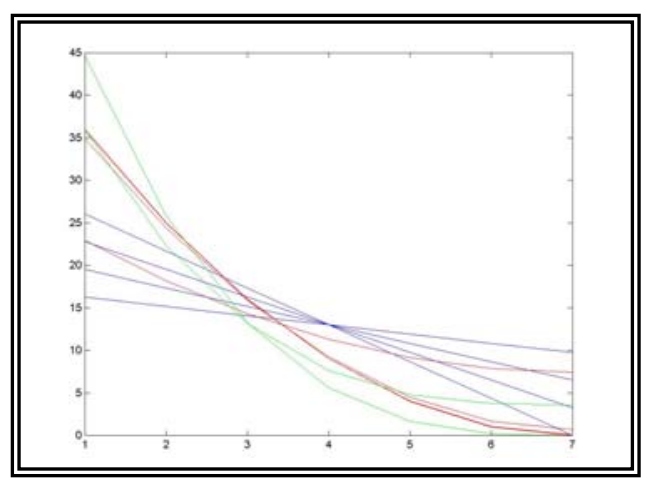

Figure 2: Variable width distribution for spiral inductor

Therefore, it can be observed that the inner diameter $\mathrm{d}_{\mathrm{in}}$, pitch (distance between to adjacent turns), length and negative pitch (distance between two turn on opposite ends) are changed and needs to be recalculated based on the changing width of each turn. However, the spacing is maintained constant between all the turns. Figure 3 shows the assigned symbols to the modified inductor.



Figure 3: Variable Width Spiral Inductor.

Similarly, for comparison, the design parameters from [1] are used again. Further verification is also carried out using HPADS momentum to determine the accuracy of the circuit approach. Tables $2 \& 3$ show the results obtained from Circuit \& HPADS simulation respectively.

\begin{tabular}{|l|c|c|c|}
\hline Circuit model & Q-factor & $\mathrm{Ls}(\mathrm{nH})$ & Freq $(\mathrm{GHz})$ \\
\hline Constant & 4.0919 & 7.3627 & 2.07 \\
\hline OptimizeQ & 5.1941 & 4.3925 & 2.835 \\
\hline OptimizeL & 4.2207 & 7.0023 & 2.16 \\
\hline & & & \\
\hline Circuit model & dout $(\mu \mathrm{m})$ & power & y-intercept \\
\hline Constant & 300 & 1 & 4 \\
\hline OptimizeQ & 276 & 1 & 1.1 \\
\hline OptimizeL & 295 & 1.9 & 3.6 \\
\hline
\end{tabular}

Table 2: Simulation results for uniform \& Variable width Spiral Inductor.

\begin{tabular}{|l|c|c|c|}
\hline HPADS & Q-factor & Ls $(\mathrm{nH})$ & Freq $(\mathrm{GHz})$ \\
\hline Constant & 3.992 & 8.617 & 1.350 \\
\hline OptimizeQ & 4.963 & 4.510 & 2.403 \\
\hline OptimizeL & 4.088 & 8.098 & 1.402 \\
\hline & & & \\
\hline HPADS & dout $(\mu \mathrm{m})$ & power & y-intercept \\
\hline Constant & 300 & 1 & 4 \\
\hline OptimizeQ & 276 & 1 & 1.1 \\
\hline OptimizeL & 295 & 1.9 & 3.6 \\
\hline
\end{tabular}

Table 3: HPADS simulation results for uniform \& Variable width Spiral Inductor.

OptimizeQ results represent solely Q-factor optimization and OptimizeL represent Q-factor optimization with an arbitrary minimum inductance value (Ls). It is observed that the changes from Circuit to HPADS simulations are consistent with that of the uniform spiral inductor. From the tables, OptimizeQ display a significant improvement of $26.9 \%$ and $24.3 \%$ for Circuit \& HPADS simulations respectively from the uniform to the variable width design. In addition, there is a reduction of an area of $8 \%$. However, this is 
accompanied by a tradeoff between Q-factor and the inductance value.

On the other hand, OptimizeL result is to show that even if a desirable inductance value is to be maintained (Ls $=7 \mathrm{nH}$ in this case), the circuit could possibly produce a design that requires smaller area. From the two tables, it can be observed that OptimizeL has produced a design with a smaller area (1.7\% reduction) and also higher Q-factor (3.1\% increase). Figures 4-6 show Circuit and HPADS simulation results of Qfactor vs. Frequency for OptimizeQ and OptimizeL respectively.

\section{CONCLUSION}

It can be observed that the inductance value for all the three results differs. Inductance value is $8-\mathrm{nH}$ for the reference paper [1], 7.3627-nH for Circuit simulation and 8.617-nH for HPADS simulation. This difference might be due to approximations and the methods used in calculation. Momentum approaches the geometry from a microscopic view while the other approaches from a macroscopic view thus resulting in different approximations. The difference of center frequency between analytical model and Momentum may be due to the lack of inter-turns capacitance calculation and the exclusion of underpass metallization thickness in Circuit simulation.

However, the simulation code has been checked for consistency and accuracy by comparing with results from that of reported literature [1]. In addition, the optimization program developed is able to produce a design that can achieve higher Q-factor than the uniform spiral inductor design. Dimension coordinates are also calculated in the process thus enabling easy verifications and fabrication. The amount of simulation time is also reduced significantly as the process is not random. With a set of specified parameters, a design with higher Q-factor and smaller area can be achieved.

\section{REFERENCES}

[1] C. Patrick Yue and S. Simon Wong, "Physical Modeling of Spiral Inductors on Silicon." March 2000, IEEE Transaction on Electron Devices, vol. 47 , no. 3 , pp. $560-568$.

[2] F. W. Grover, Inductance Calculations. 1962, New York, NY: Van Nostrand.

[3] H. M. Greenhouse, "Design of planar rectangular microelectronic inductors." June 1974, IEEE Trans. Parts, Hybrids, Pack, vol. PHP-10, pp. 101-109.

[4] Lopez-Villegas et al., "Improvement of the quality factor of RF integrated inductors by layout optimization." 1998, IEEE Radio Frequency Integrated Circuits (RFIC) Symposium, pp.169-172.

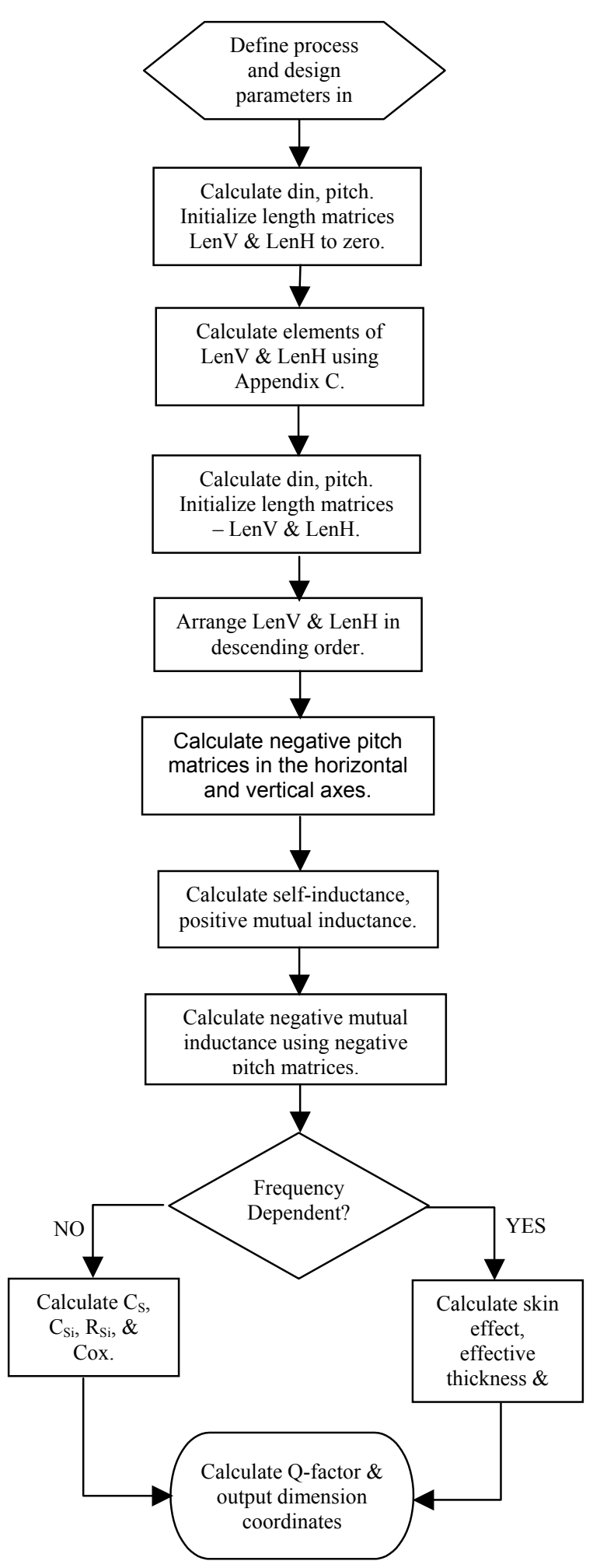

Flowchart 1: Calculation of uniform spiral inductor's $Q$-factor \& inductance. 


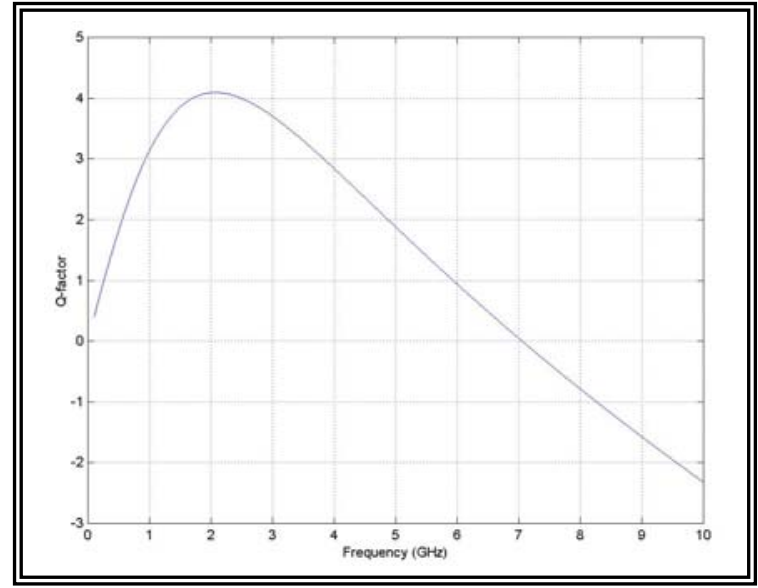

(a)

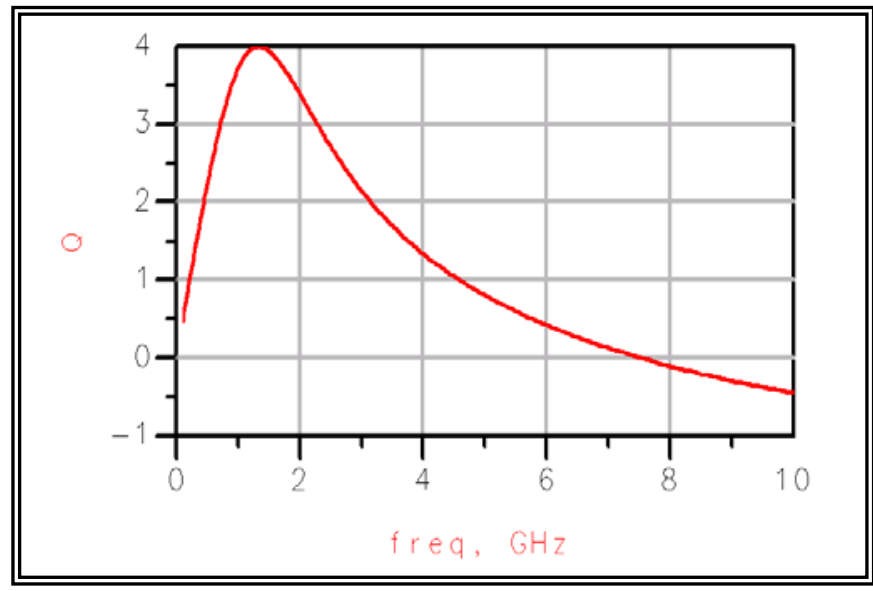

(b)

Figure 4: Q-factor vs. Frequency uniform spiral inductor. (a) Circuit simulation. (b) HPADS simulation.

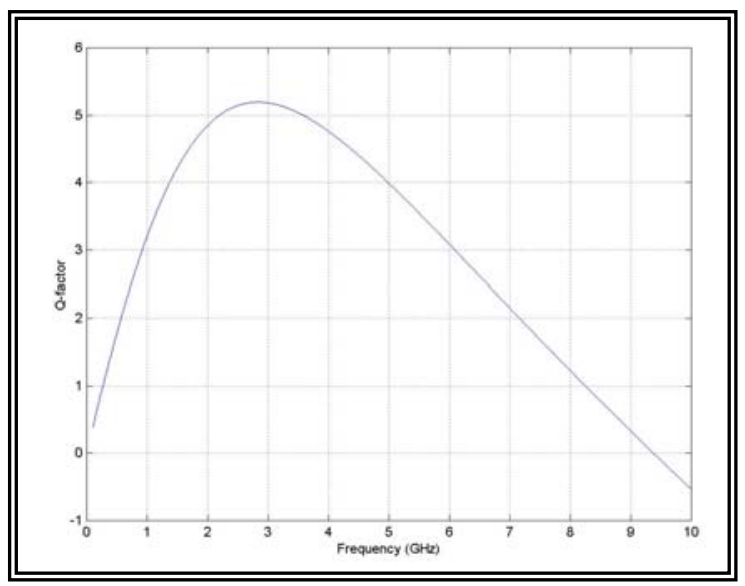

(a)

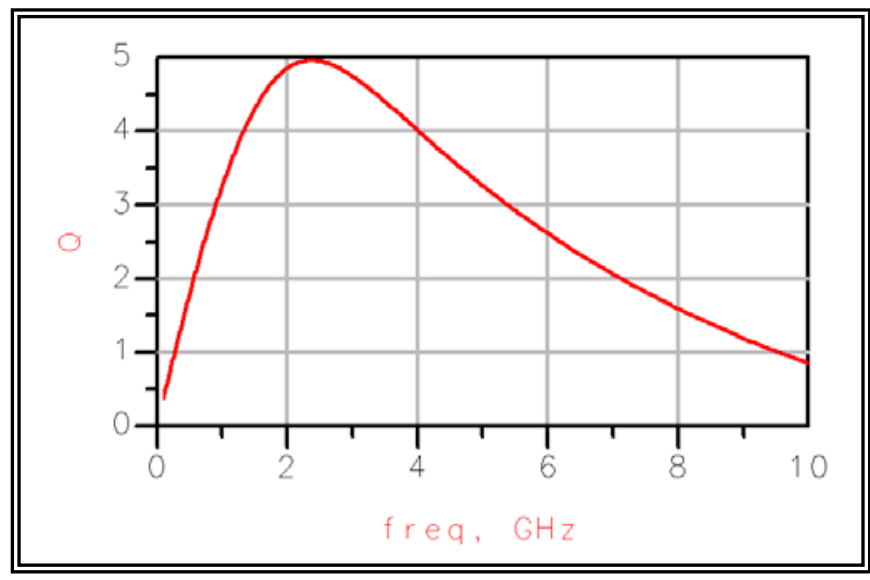

(b)

Figure 5: Q-factor vs. Frequency for OptimizeQ. (a) Circuit simulation. (b) HPADS simulation.

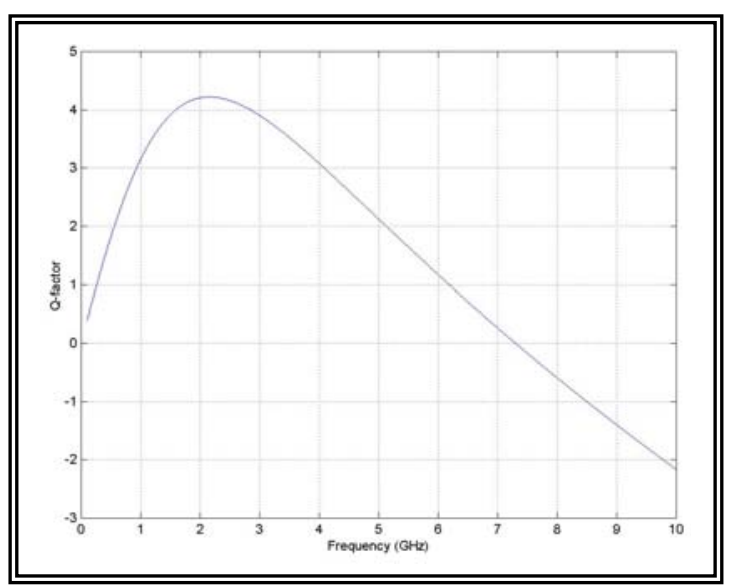

(a)

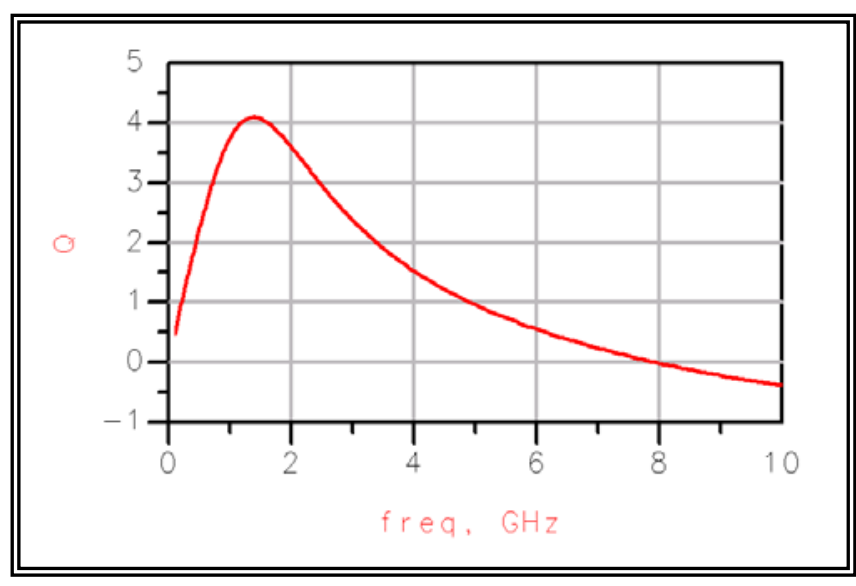

(b)

Figure 6: Q-factor vs. Frequency for OptimizeL. (a) Circuit simulation. (b) HPADS simulation. 